\title{
Éxito cognitivo y virtud extendida
}

\section{Cognitive Success and Extended Virtue}

\author{
RODRIGO LAERA \\ CONICET - UNIVERSIDAD DE BARCELONA
}

Recibido: 15/03/2019 Aceptado:20/03/2019

\begin{abstract}
RESUMEN
El objetivo del presente trabajo consiste en presentar la noción de virtud extendida considerando tres aspectos fundamentales de éxito cognitivo (i) el carácter dinámico de la recolección y evaluación de evidencias; (ii) la fiabilidad de los procesos de predicción; (iii) la aptitud para tratar con un contexto dado, donde las capacidades extendidas se integran con las no extendidas. En este sentido, el éxito cognitivo es producto de una función entre la recolección de evidencias y su costo en tiempo y esfuerzo. La virtud extendida tiene como fin reducir dicho costo.

PALABRAS CLAVE

MENTE EXTENDIDA, VIRTUD EPISTÉMICA, ÉXITO COGNITIVO, EXTERNISMO
\end{abstract}

\section{ABSTRACT}

The aim of this paper is to present the idea of virtue extended under three fundamental aspects of cognitive success (i) the dynamic nature of the collection and evaluation of evidence; (ii) the reliability of the prediction processes; (iii) the ability to deal with a given context, where extended capabilities are integrated with non-extended ones. In this sense, cognitive success is the product of a function between the collection of evidence and its cost in time and effort. The extended virtue is intended to reduce said cost.

KEYWORDS

EXTENDED MIND, EPISTEMIC VIRTUE, COGNITIVE SUCCESS, EXTERNALISM

(C) Contrastes. Revista Internacional de Filosofía, vol. XXIV-Nº3 (2019), pp. 59-72. ISSN: 1136-4076

Departamento de Filosofía, Universidad de Málaga, Facultad de Filosofía y Letras Campus de Teatinos, E-29071 Málaga (España) 


\section{I.INTRODUCCIÓN}

A GRANDES RASGOS, LA TESIS DE LA MENTE EXTENDIDA sostiene que los procesos cognitivos que componen la mente humana pueden ir más allá de los límites del individuo para incluir como partes apropiadas aspectos del entorno físico y sociocultural. A su vez esta idea puede conectarse con la tesis de la cognición extendida para terminar por sostener que las virtudes específicamente epistémicas pueden depender de sistemas cuyos límites no son los del cerebro o los del organismo biológico, como ya ha propuesto Prichard (2010). No obstante, recientemente, Clark (2015) ha cuestionado esta conexión, sugiriendo que la epistemología de la virtud se identifica principalmente con las facultades cognitivas confiables, como la percepción, la memoria y el pensamiento racional, cuyo eje principal es el sujeto ( $c f r$., Sosa 2007). En consecuencia, si la adquisición de conocimiento pertenece al carácter intelectual del agente, entonces las herramientas tecnológicas no pueden llegar a concebirse como un elemento no biológico de la maquinaria de la mente. El primer objetivo de este trabajo consiste en exponer la idea de que la noción de mente extendida no es incompatible con una variante epistémica de virtud que tenga como eje tres aspectos fundamentales del éxito cognitivo (i) el carácter dinámico de la recolección y evaluación de evidencias; (ii) la fiabilidad de los procesos de predicción; (iii) la aptitud para tratar con un contexto dado, donde las capacidades extendidas se integran con las no extendidas. El segundo objetivo consiste en presentar la hipótesis de la virtud extendida sobre la base de una función que relaciona la recolección de evidencias con su costo en tiempo y esfuerzo para alcanzar cierto éxito cognitivo. Alcanzar ambos objetivos implica abandonar el proyecto de la epistemología tradicional, en primera persona.

\section{VIRTUDES EPISTÉMICAS COMO CARTESIANAS}

A partir de los trabajos de Prichard (2010); Palermos \& Pritchard (2013) y Clark (2015), la hipótesis de la mente extendida ha entrado en el foco de la epistemología, especialmente de la epistemología de la virtud, enfrentándose a una de sus tesis fundamentales: que la adquisición de conocimiento, a fin de cuentas, pertenece al carácter intelectual del agente. Tomemos dos definiciones, que ya son clásicas, de virtud epistémica como una especie de muestra:

(i) $S$ tiene una virtud intelectual o facultad relativa a un entorno, si y solo si tiene una naturaleza interna a partir de la cual la mayoría de las veces conduce a la verdad y evita errores en ciertos campos de proposiciones y en ciertas condiciones (ej., Sosa, 1991). 
(ii) Las virtudes intelectuales son facultades innatas o hábitos adquiridos del agente, que hace posible que una persona arribe a la verdad y evite el error en algún campo relevante (ej., Greco, 2002).

Dejando de lado los aspectos problemáticos de ambas definiciones; sobre todo los que se refieren al objetivo de alcanzar la verdad o evitar el error (además de los problemas derivados de las condiciones de probabilidad que implica la idea de «la mayoría de las veces» o la noción misma de «posibilidad»), lo que importa destacar es que ambas definiciones se centran en una habilidad subjetiva de aportar creencias fiables. Mediante una división categórica entre lo interno y lo externo al individuo, el conocimiento se entiende como un caso especial de desempeño meritorio del agente. Las dos definiciones proponen una epistemología en primera persona que se enmarca dentro del proyecto cartesiano, como ya ha sostenido Kvanvig (1992). El agente posee capacidades o facultades internas sobre creencias particulares que se articulan con datos externos para sostener que conoce o sabe que $p$, incluso para cualquier creencia correcta, la corrección es atribuible a una competencia que se deriva un ejercicio en condiciones adecuadas. Aunque ciertos tipos de estados mentales intencionales, como las creencias y los deseos, deban estar necesariamente relacionados con el medio ambiente de la manera correcta para intervenir en el desempeño intelectual del agente, persiste un afuera externo al cerebro y un adentro interno al cerebro, donde el proceso de formación de las creencias, que cuenta como conocimiento conducente, ha sido procesado por determinadas funciones cognitivas que el agente posee como algo que le es propio.

Ahora bien, si la dicotomía entre lo interno y lo externo se desarrolla en términos de creencias particulares fundadas por virtudes intelectuales en el cerebro del agente, entonces las herramientas tecnológicas no se entenderán como elementos no biológicos de la maquinaria de la mente. Dicho de otra manera, si el carácter cognitivo reside en el propio organismo, en sus capacidades, como recuerdos o percepciones, entonces los procesos que están fuera de los límites del cerebro solamente integrarán el conjunto o caja de herramientas disponibles subordinadas a las capacidades internas de los agentes. Se podrá hablar de «habilidades adquiridas»; de «métodos de investigación adquiridos» o «entrenamiento especializado» o incluso «tecnología avanzada integrada», pero este tipo de expresiones solamente tendrán sentido desde la perspectiva de la epistemología de la virtud como parte del dominio de un agente con tales capacidades, un núcleo cognitivo ejecutor centrado en las propiedades internas de la mente.

Visto de esta manera, el diseño de una epistemología de la virtud en primera persona contrasta con la hipótesis de la mente extendida ¿pero es esto necesariamente así? ¿Puede haber una interpretación en la que sean 
compatibles? ¿Puede la epistemología de la virtud romper con el proyecto cartesiano? ¿Se puede pensar en algo así como en una virtud extendida?

\section{VIRTUD EXTENDIDA}

Resulta interesante comparar la noción de virtud epistémica con la tesis de la mente extendida; pues si se acepta, entonces es posible proponer un modelo de virtud extendida que entienda a los procesos intelectuales como una parte más de la interacción humana con el mundo. Por ejemplo, de la misma manera que la capacidad intelectual de pensar juzgadas en el ajedrez puede considerarse como parte del juego - una acción que le corresponde al juego-; las anotaciones que un agente hace en su diario puede considerarse como una especie de memoria, es decir como parte de los procesos de almacenación y recuperación de la información que el propio agente dispone. Una idea antigua que incluso aparece en el Fedro de Platón, donde Sócrates considera a la escritura como una especie de memoria artificial, un remedio para el olvido. Pero no solamente para nuestro olvido, sino también para el olvido en los demás.

La idea general es que los recursos tecnológicos se han enredado tan profusamente con nuestra maquinaria biológica que se han integrado a los procesos cognitivos, siendo también parte de nuestras virtudes epistémicas. Siguiendo Clark \& Chalmers (1998), si, al realizar alguna tarea, una parte del mundo funciona como un proceso que, si continuara en la cabeza, no dudaríamos en reconocer como parte del proceso cognitivo, entonces esa parte del mundo es parte del proceso cognitivo. ${ }^{1}$ De lo que se desprende que se considera un mero prejuicio suponer que toda cognición deba tener lugar dentro de los límites de la piel y el cráneo del organismo, o deban ser estrictamente biológicos. Así, la teoría de la virtud epistémica no debería preocuparse solo por los procesos que tienen lugar dentro de los agentes cognitivos cartesianos, también debe abarcar, en una especie de pie de igualdad, la articulación entre el carácter interno a la piel del agente y al cráneo y el entorno físico y sociocultural. En consecuencia, la virtud epistémica se expande hacia una forma interactiva de los recursos externos, conformando lo que se podría llamar «virtud epistémica extendida» 0 , simplemente, «virtud extendida» (VE).

La VE puede caracterizarse, a grandes rasgos, como aquella que sostiene que las virtudes de los agentes no están concentradas en las capacidades subjetivas, sino que conforman un conjunto de habilidades que exceden la dicotomía epistémica entre lo interno y lo externo, incorporando herramientas que en el origen filogenético no estaban presentes. De manera que el éxito

1 Clark \& Chalmers llaman a esta afirmación «tesis de paridad». Para una discusión a fondo de esta tesis, se puede consultar, por ejemplo: Menary (2007), Sprevak (2009), Rupert (2009). 
cognitivo sería una relación entre dichas capacidades y sus logros epistémicos. Para esto, los agentes no necesitan racionalizar los diversos procesos que tienen lugar afuera del cerebro y que participan de la organización de recursos disponibles, sino aprender a manejarlos explotando sus costes y beneficios.

Siguiendo esta línea teórica, la noción de mente extendida parece compatible con esta variante epistémica de la virtud. En este sentido, importa señalar tres ejes fundamentales del éxito cognitivo en la hipótesis señalada de la VE: (i) el carácter dinámico de la recolección y evaluación de evidencias; (ii) la fiabilidad de los procesos de predicción; (iii) la aptitud para tratar con un contexto dado, donde las capacidades extendidas se integran con las no extendidas.

Respecto al primer eje (i), la teoría de la virtud puede articularse con la hipótesis de la mente extendida cuando se buscan evidencias en las que apoyar una creencia. El desarrollo tecnológico ha puesto a la mano un mecanismo por el cual se pueden encontrar evidencias más rápidamente que sin él, y esto ya formaría parte de las habilidades que organizan nuestra forma de conocer. De este modo, es posible pensar que determinadas evidencias se recogen únicamente mediante mecanismos tecnológicos integrados a nuestras habilidades intelectuales, como parte de ellas, eludiendo el carácter sincrónico y personalista de la virtud.

Respecto al segundo eje (ii) la tesis de la virtud epistémica puede interpretarse de manera fiabilista y con ello tender un puente a la hipótesis de la mente extendida. Sin entrar en los distintos tipos de fiabilismo y siguiendo la línea de este trabajo, la idea sería que un agente es cognitivamente exitoso cuando su virtud epistémica se besa en un proceso fiable, que predice estados de cosas, formado por un cuerpo de evidencias disponibles que soporten sus creencias. En efecto, si los procesos fiables son parte de nuestras habilidades cognitivas, que incluyen evidencias recogidas por los agentes, entonces cabe preguntar qué conforma un proceso fiable y si es necesario reconocerlo como tal. Si nos guiamos por la idea de virtud epistémica tradicional, el agente poseerá las habilidades intelectuales necesarias para distinguir los procesos fiables de los no-fiables, los objetos o estados de cosas que conforman un cuerpo de evidencias de los que no. Pero si la virtud epistémica se articula con el fiabilismo y la hipótesis de la mente extendida, entonces el proceso fiable estará enfocado de manera distinta. Por un lado, el proyecto de una virtud epistémicamente extendida podría ser enmarcado dentro de la epistemología social de índole extensiva (en el sentido de Goldman, 2010): el de un campo interdisciplinario en el que las dimensiones sociales de la adquisición de conocimiento y el tratamiento de la información mediante tecnología se encuentran enraizados en la fiabilidad de los procesos cognitivos. Por otro lado, el proyecto no tendrá nada que decir acerca del debate entre el internismo y 
el externismo epistémico, aunque presumiblemente asumiría un enfoque antiindividualista. Si se acepta que el éxito cognitivo depende de dimensiones sociales y tecnológicas, entonces no hay razón de principio para negar que los procesos cognitivos puedan distribuirse de manera que nuestros cerebros sean interdependientes de los artefactos o de los otros agentes con los que interactuamos. ${ }^{2}$

Respecto al tercer eje (iii), se puede pensar que al hablar de capacidades extendidas, implícitamente uno está aceptando capacidades no extendidas y con ellas volver al paradigma sujeto-objeto, delineado desde la tradición filosófica como una orientación desde adentro hacia afuera o desde afuera hacia dentro del cuerpo biológico del agente. Sin embargo, importa aclarar que la noción extendida de la virtud epistémica no implica negar la importancia del cerebro en utilización de evidencias, ni que las habilidades intelectuales amplíen procesamiento cognitivo. En todo caso, lo que se pretende examinar es cómo, en el contexto de un organismo activo y cognitivamente exitoso, cierto cuerpo de cosas mundanas podría considerarse la base de la realización de nuestras capacidades epistémicas. Nada en este marco teórico hace suponer que el cerebro sea incapaz de explorar el mundo por sí solo o que las tecnologías sean parte de él.

\section{IV. ÉxITO COGNITIVO}

Habitualmente la tesis de la virtud epistémica tradicional debe enfrentar el problema de dispersión, que consiste en la necesidad presentar un creciente cuerpo de principios epistémicos para explicar la justificación de creencias encarnadas en agentes de muy distintos tipos. Por ejemplo, suponer que seres extraterrestres tengan habilidades completamente diferentes de las nuestras y usar tales capacidades para adquirir conocimiento. Este problema puede ampliarse si consideramos que la virtud sea extendida. El problema en el que nos encontraríamos sería el de establecer una diferencia clara entre mundo circundante o entorno y la capacidad cognitiva como un todo: cuál es una propiedad cognitiva, cuál no lo es y cómo separarlas. Dicho de otra manera, ¿qué criterio de demarcación poseemos para delimitar propiedades extendidas de la virtud epistémica de aquellas propiedades que pertenecen al mundo circundante?

2 Esto se puede confrontar con el problema de la generalidad. Este problema sostiene que creencia es causada por una secuencia de eventos particulares que es una instancia de muchos tipos de los procesos causales, lo que conduce a la confusión en la noción misma de fiabilidad que es una propiedad de los tipos de procesos de formación de creencias, no de secuencias de acontecimientos particulares. 
Llegados a este punto, se puede proponer una hipótesis de VE apoyada en la recolección de evidencias para la justificación de una creencia de cualquier tipo. La hipótesis presenta dos supuestos: (i) que las evidencias recogidas tienen la misma fuerza probatoria, es decir que se comportan de la misma manera con respecto a la justificación, así a mayor cantidad de evidencia, mayor es nuestro éxito cognitivo; (ii) que la virtud epistémica radica solamente en capturar esas evidencias. El primer supuesto es mucho más fuerte que el segundo, y puede ser completamente inaceptable en algunos casos, pero dada las pretensiones de este trabajo parece apropiado.

Dicho esto, no resulta complicado aceptar que el éxito cognitivo es el resultado de una función entre la cantidad de evidencias recogidas y la virtud epistémica de conseguirlas, tal virtud incluye el esfuerzo y el tiempo como un factor decisivo, así como la pericia, la adecuación al entorno y, cómo no, circunstancias aleatorias que son azarosas. De esta manera, cuanto más esfuerzo epistémico, mayor cantidad de evidencias, con lo que se va mejorando la relación entre la virtud y su alcance en evidencias. Mayor alcance, más éxito cognitivo. Por el contrario, menor esfuerzo, menor cantidad de evidencias recogidas, menor éxito cognitivo. Lo podemos ilustrar de la siguiente manera:

- Curva de éxito cognitivo.

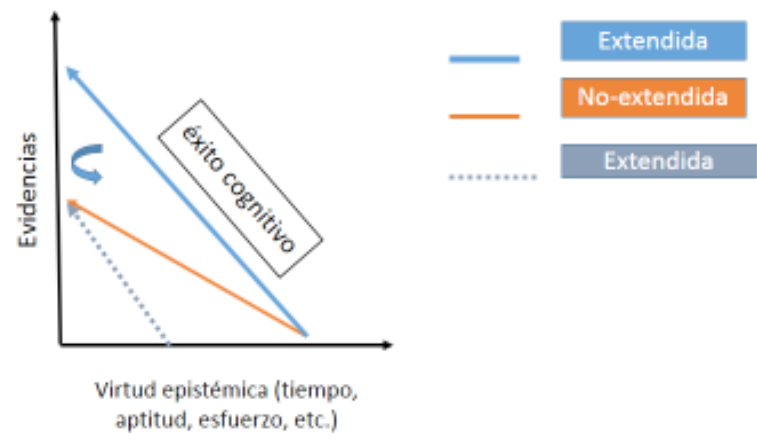

Ahora bien, cuando la virtud se hace extendida, cuando se incorpora en ella herramientas tecnológicas que disminuyen la cantidad de tiempo, 
esfuerzo o producen una mejora en la aptitud, entonces la pendiente del éxito cognitivo cambia, haciéndose más inclinada, más vertical. Mientras que el mundo circundante no incorporado a la VE no modificará la pendiente. Dicho de otra manera, la virtud es extendida cuando la pendiente del éxito cognitivo se encuentre inclinada de tal manera que no lo estaría si la virtud no estuviera extendida. Nuevamente, el criterio expuesto consiste en señalar que cuando el mundo circundante no inclina la curva del éxito cognitivo, entonces no forma parte de la virtud extendida o no extendida. Esto permite reconocer que, partiendo del punto de vista fiabilista, la producción de evidencias mejora al optimizar la calidad del procesamiento intelectual incorporando aspectos transformadores del entorno al cuerpo del agente. Esto es justamente lo que señala la línea punteada. La mejoría de la calidad pasa entonces por el hecho de ahorrar tiempo y esfuerzo. ${ }^{3}$

Por supuesto, los agentes pueden ser más o menos sensibles aun extendiendo su virtud y esto será el lugar de la responsabilidad en del éxito cognitivo. En la tradicional teoría de la virtud la responsabilidad permitía valorar las habilidades del agente valorando sus aptitudes en lugar de simplemente valorar sus consecuencias. No era lo mismo que el agente calcule mentalmente una cuenta matemática a que se use una calculadora. En el primer caso había mérito epistémico gracias al funcionamiento de sus capacidades intelectuales, mientras que en el segundo no. Ahora bien, si los procesos externos se integran mediante la VE, la capacidad intelectual del agente puede desplazarse hacia un nivel de vigilancia y monitoreo o a un nivel ejecutor y evaluador. Esto implica una especialización del aparato cognitivo y un mejor funcionamiento en ese nivel. Los agentes podrían maximizar sus recursos dedicándose intelectualmente al monitoreo del correcto funcionamiento de sus habilidades cognitivas para dejar a su parte extendida el proceso y adquisición de evidencias. Así, el repertorio de las posibilidades epistémicas de los agentes no solamente podría ampliarse, sino especializarse. A medida que la virtud epistémica introduce extensiones, tales extensiones pueden ser cada vez más sofisticadas y más específicas hasta alcanzar recursos de un solo usuario, de manera uno puede dejar a la parte no extendida con las actividades genuinamente colectivas. ${ }^{4}$

Dos cuestiones más. La primera se refiere a lo siguiente: ¿la curva de éxito cognitivo se puede desplazar hacia abajo producto de la virtud extendida? Si este fuera el caso, ocurriría que obtendríamos menos evidencias a partir de la virtud extendida que sin extenderla. Supóngase que $S$ tiene una evidencia

3 Vale aclarar que esto podría estar enmarcado dentro de un proyecto post-conitivista o funcionalista de las atribuciones mentales (cf. Burdman, 2015).

4 Para un desarrollo entre extensiones individuales y colectivas, véase: Sterelny (2010) 
que apoya su creencia $p$ sin que la virtud intelectual de su hallazgo no fuera extendida, lo que puede interpretarse como un éxito cognitivo por parte de $S$. Ahora bien, tras el desarrollo de una tecnología, que se incorpora en las capacidades intelectuales de $S$ de manera constitutiva, surge un nuevo escenario. En este nuevo escenario $S$ descubre que su evidencia era falsa, de modo que aquello que se interpretaba como éxito cognitivo en realidad no lo era. Es decir, había obtenido un falso positivo. No obstante, a partir de la extensión virtuosa, $S$ descubre que la creencia en $p$ es más compleja de lo que parecía en el momento no extendido, y que necesita más recursos para conseguir evidencias, sin caer en un nuevo falso positivo. En este tipo de casos, se podría pensar que la curva de éxito cognitivo se movió hacia abajo y que su inclinación se hizo más plana. Pero lo que ha sucedido es que se cambió una trayectoria aparente de éxito cognitivo (en función de evidencias aparentes) por una trayectoria real. Este es el cambio que sucede cuando se descubren falsos positivos.

La segunda cuestión nace de la primera, a saber: ¿puede la virtud extendida conceder un falso positivo? En este caso la curva del éxito cognitivo resulta de evidencias conseguidas a partir de VE. Aquí cabría suponer que, si el conocimiento es falible, entonces el conocimiento brindado por la extensión de la virtud también puede ser falible. Esto lleva a considerar que si una virtud no extendida puede supervisar a la extendida con el fin de evitar fallos, entonces ambas deben estar adecuadas para formar un todo epistémico. Por ejemplo, $S$ tiene la creencia de que $p$, apoyado en evidencias fundadas en la extensión de la virtud. Pero, mediante la supervisión de las evidencias a través de recursos intelectuales no extendidos, se llega a considerar que son falsas a la luz de nuevas evidencias conseguidas mediante virtudes no extendidas. En este caso también se estaría en presencia del falso positivo y los recursos tecnológicos integrados serían su causa. Así, la integración no es dependencia: que los recursos tecnológicos estén integrados, no significa que VE dependa únicamente de ellos.

Por último, el éxito cognitivo es el mismo al margen de si los procesos para recoger evidencias se encuentran dentro o fuera del cerebro; de modo que la virtud extendida no trata con un mero problema de locación, pues no existe una ubicación incorrecta que anule la obtención de evidencias. La extensión de la virtud epistémica se caracteriza como una manera de optimizar recursos para conseguir más y mejores evidencias, aunque pueda suceder que en alguna ocasión haya algún falso positivo.

\section{DOS OBJECIONES Y RESPUESTAS}

A partir de lo expuesto en los apartados anteriores, se pueden presentar dos objeciones elementales. La primera objeción ataca la relación entre extensión 
y éxito cognitivo, basándose en que la epistemología extendida no especifica el rol de interacción con la tecnología/mundo circundante, sin considerar un núcleo cognitivo ejecutor virtuoso. La segunda objeción se dirige a la noción de poco intuitiva de virtud intelectual en contraposición con la extendida, pues anula la intuición acerca del mérito del agente cuando descubre una nueva evidencia. ${ }^{5}$

\section{Primera objeción.}

Cuando se sostiene que las capacidades extendidas pueden integrarse con las no extendidas de la virtud epistémica, se puede pensar que la dependencia de los elementos externos para algunas formas de conocimiento no es una razón suficiente que demuestre la participación constitutiva del exterior en el fenómeno mismo de cognición. ${ }^{6}$ Por ejemplo, respecto a los casos simples de conocimiento perceptivo, que no está dentro del control inmediato de uno, diversas partes del entorno son necesarias como parte del éxito cognitivo; sin embargo, no por eso se afirma que son constitutivos a las capacidades extendidas del cerebro. En casos tales como el conocimiento perceptivo, donde se articulan los datos externos con las habilidades intelectuales, no sería posible separar entre lo que es accesorio al conocimiento de lo que es constitutivo. Por lo tanto, se puede decir que si los objetos de la percepción no estuvieran, entonces la obtención de evidencias sería distinta, y sería excesivo señalar a todos los objetos de la percepción forman parte de las virtudes extendidas.

Cuando se trata de algún tipo de interacción tecnológica, esta debería ser separada del entorno externo. Pero solamente es posible tal separación con un núcleo ejecutor que, finalmente, resulte intelectualmente virtuoso. Aceptar esto es reconocer que los agentes utilizan herramientas eficaces para alcanzar el éxito cognitivo, pero que estas herramientas no constituyen el núcleo ejecutor, sino que están gracias a él. Así, las herramientas tecnológicas serían utilizadas por los agentes virtuosos sin ser parte de la virtud.

Respuesta.

Para elaborar una respuesta a la anterior objeción conviene tener en mente la diferencia entre el externismo activo del externismo tradicional. En el primero los estados mentales están constituidos en parte por las características del entorno, mientras que en el externismo tradicional el significado de los términos de clases naturales se determina por el entorno natural o social. ${ }^{7}$

5 Se dejan de lado las objeciones metodológicas, ya que exceden al marco teórico de este trabajo.

6 Véase para esta crítica: Adams y Aizawa (2008) o Rupert (2010)

7 Para un ejemplo de externismo activo, véase: Clark y Chalmers (1998). Para un caso de externismo tradicional, véase: Putnam (1975); Burge (1986), para quienes estudiar el cerebro, aislado de su entorno tendría resultados incompletos. 
La tesis de la VE se refiere a un tipo de externismo activo, en el que parte del entorno aumenta los recursos computacionales del agente con el fin de alcanzar el éxito cognitivo. Este segmento del entorno se encuentra integrado a las habilidades intelectuales del agente, de manera que la obtención de evidencias sería diferente si no estuviera, lo que excede a encapsularse en las habilidades subjetivas de aportar evidencias. Ahora bien, hay tecnologías que ayudan a capturar mejor evidencias perceptuales, por ejemplo, algo tan simple como un par de anteojos, y otras tecnologías que son imprescindibles para capturarlas, por ejemplo un telescopio. Respecto a esto uno puede optar por considerar al conocimiento como siendo encarnado (embodied); situado; o extendido, La primera opción sostiene que la mente existe en todo el cuerpo, no solamente en el sistema nervioso central. La segunda sostiene que hay antecedentes ambientales o sociales que son necesarios para adquirir ciertas funciones cognitivas. La tercera opción radica en que el cerebro con el cuerpo $\mathrm{y}$ el mundo conforman un acoplamiento dinámico $\mathrm{y}$, por lo tanto, estados mentales y cognitivos. Esta última opción, no anula las dos anteriores, sino que las implica. Y es justamente este aspecto dinámico en relación a la obtención de evidencias lo que distingue a la hipótesis de la VE de la virtud epistémica tradicional. En este sentido, los agentes epistémicos están contenidos en el medio ambiente, que es algo más que el mero fondo desde donde recoger evidencias. Si bien las percepciones son, prima facie, imprescindibles a nuestro conocimiento, ellas por sí solas no corren la curva del éxito cognitivo, sino que son su posibilidad. Asimismo, la VE no es una hipótesis sobre la posibilidad del conocimiento, sino sobre las herramientas que se encuentran integradas al sistema para poder recoger más y mejores evidencia, aún a pesar de los falsos positivos.

Segunda objeción.

Una de las características elementales de la virtud epistémica consiste en poner un gran énfasis en el mérito de los agentes al obtener conocimiento. No obstante, si no hay una auténtica distinción entre aquellas propiedades intelectuales que son intrínsecas al agente y aquellas que no lo son, entonces el desempeño meritorio de obtener conocimiento se diluye dentro de cualquier forma de éxito cognitivo. Esto no solamente confronta con de la idea de virtud en sí misma, sino también del carácter intuitivo acerca de cómo funciona el desempeño epistémico de los agentes.

Supongamos el seeiguiente caso. En un mundo de virtudes extendidas, Ana es una buena alumna, porque posee un uso óptimo de la calculadora. Mediante el buen uso de la calculadora, ella resuelve correctamente tanto ecuaciones simples como complejas. Dado que, en este mundo de virtudes extendidas, el mérito del agente carece de importancia, se nos permite afirmar que Ana sabiendo usar la calculadora, sabe matemáticas. Poco importa, puesto 
que logra integrar la calculadora en su sistema cognitivo, si sabe sumar por si misma o no. En el mundo de virtudes extendidas, enseñar matemáticas es enseñar a usar la calculadora. Todo el mérito epistémico se reduce simplemente a eso.

Respuesta.

Cuando se incrementa y mejora la calidad de las evidencias que se encuentran disponibles mediante el uso de la tecnología integrada, los agentes epistémicos pueden desligarse del compromiso de conocer los detalles de los procesos en vistas a beneficios más elevados o a costos menores. Esto puede entenderse como una mayor cantidad de esfuerzo y tiempo disponible que los agentes pueden usar para otras actividades. Esto explica las ventajas de la virtud extendida. Pero no todas son ventajas. Una desventaja es olvidar cómo se realizan de ciertos procesos. Pero este olvido puede ser también parte del éxito cognitivo. Cuando se alcanza el éxito utilizando siempre un mismo patrón, se omite la posibilidad de alcanzarlo mediante otros patrones. Uno abandona el proceso no extenso de alcanzar una meta epistémica, porque mediante el proceso extenso la alcanza más fácilmente. De la misma manera, si se empieza a alcanzar ciertas metas epistémicas mediante el uso de procesos externos, entonces es posible que se desatienda otra alternativa, en este caso no extensa, de alcanzarlas, como puede ser el caso de Ana.

Respecto al carácter intuitivo del mérito en la virtud epistémica, la VE prescinde del carácter personalista, en primera persona, de la epistemología tradicional de la virtud. Justamente, el carácter dinámico de la recolección y evaluación de evidencias y la fiabilidad de los procesos de predicción, excluyen la idea de una epistemología hipostasiada en la primera persona. Así, el carácter meritorio radica en la sensibilidad del agente para apropiarse de los recursos que hacen a la extensión de la virtud. Por lo tanto, la atribución de mérito epistémico se percibe como una categoría semántica que referencia a un todo (agente-mundo) extendido. Es más, la curva de éxito cognitivo no radica en un mérito ligado a la subjetividad de un agente, sino en el logro de coordinar correctamente aspectos bilógicos con otros no biológicos para obtener más y mejores evidencias con menor costo.

\section{CONCLUSIÓN}

La propuesta de este trabajo consistió en conectar la tesis de la cognición extendida con la noción epistémica de virtud, dando lugar a la hipótesis de virtud extendida -en la que los límites cognitivos no son los del cerebro o los del organismo biológico. Vale recalcar que esta no es una hipótesis subpersonal, por ello el éxito cognitivo puede verse como el producto de una función, donde la producción o recolección de evidencias depende del tiempo, el esfuerzo y la aptitud del agente. Las capacidades meramente intelectuales 
dan una curva determinada de éxito cognitivo; cuando estas capacidades se extienden, la curva cambia, dando lugar nuevos costes y beneficios. Esta hipótesis puede proporcionar un marco analítico interesante para repensar los distintos procesos de adquisición de conocimiento.

\section{REFERENCIAS BIBLIOGRÁFÍCAS}

ADAM, F. \& AIZAWA, K., 2008. The bounds of cognition. Oxford: Blackwell.

BURDMAN, F «El post-cognitivismo en cuestión: extensión, corporización y enactivismo». Principia 19(3), pp. 475-495.

BURGE, T., 1986. «Individualism and psychology». The Philosophical Review, 95(1), pp. 3-45.

CLARK, A., 2015. What 'extended me'knows». Synthese, 192(11), pp. 3757-3775.

CLARK, A. \& CHALMERS, D., 1998. «The extended mind». Analysis, 58(1), pp. 7-19.

GOLDMAN, A., 2010. "Why social epistemology is real epistemology». En: A. Haddock, ed. Social Epistemology. Oxford: Oxford University Press, pp. $1-28$.

GRECO, J., 2002. «Virtues in epistemology». En: P. K. Moser, ed. The Oxford handbook of epistemology. Oxford: Oxford University Press, pp. 287-315.

KVANVIG, J., 1992. The intellectual virtues and the life of the mind: On the place of the virtues in contemporary epistemology. Savage: Rowman \& Littlefield.

MENARY, R., 2007. Cognitive integration: Mind and cognition unbounded. London: Palgrave Macmillan.

PRITCHARD, D., 2010. «Cognitive ability and the extended cognition thesis». Synthese, 175(1), pp. 133-151.

PRITCHARD, D. \& PALERMOS, S., 2013. «Extended knowledge and social epistemology». Social Epistemology Review and Reply Collective, Volumen 8, pp. 105-120.

PUTNAM, H., 1975. «The Meaning of 'Meaning'». Language, mind, and knowledge. Minnesota studies in the philosophy of science, Volumen 7, pp. 131193.

RUPERT, R., 2009. Cognitive Systems and the Extended Mind. Oxford: Oxford University Press.

RUPERT, R., 2010. «Extended cognition and the priority of cognitive systems». Cognitive Systems Research, 11(4), pp. 343-356.

SOSA, E., 1991. Knowledge in Perspective: Selected Essays in Epistemology. Cambridge: Cambridge University Press.

SOSA, E., 2007. A Virtue Epistemology: Apt Belief and Reflective Knowledge, Vol. I. New York: Oxford University Press.

SPREVAK, M., 2009. «Extended cognition and functionalism». The Journal of Philosophy, 106(9), pp. 503-527.

STERELNY, K., 2010. «Minds: extended or scaffolded?»Phenomenology and the Cognitive Sciences, 9(4), p. 465-481. 
Rodrigo LAERA es investigador del Consejo Nacional de Investigaciones Científicas y Técnicas (Argentina).

Lineas de Investigación:

Epistemología social y Teorías de la razón

Publicaciones recientes:

(2019), «Relativism in Context». Aufklärung 6(1), pp. 11-24.

(2018), «The Economic Mathematization: A Bibliometric Analysis». Atlantic Review of Economics 1(3), pp. 1-15.

Correo electrónico: rodrigolaera@gmail.com 